\title{
A Supervised Hybrid Methodology for Pose and Illumination Invariant 3D Face Recognition
}

\author{
Nita M. Thakare \\ Assistant Professor, \\ Dept.. of C.T. \\ P.C.E. Nagpur, India
}

\author{
V.M. Thakare \\ Professor, \\ Dept. of CSE \\ S.G.B. University, Amravati, India
}

\begin{abstract}
The 2D face recognition systems encounter difficulties in recognizing faces with illumination variations. The depth map of the 3D face data has the potential to handle the variation in illumination of face images. The view variations are handled by using the moment invariants. Moment Invariants are used as rotation invariant features of the face image. For feature matching an efficient fuzzy-neural technique is proposed. The PCA components of normalized depth map and Moment invariants on mesh images are used successfully to implement a fuzzy neural network based fully automatic 3D face recognition system. The system is evaluated on the $3 \mathrm{D}$ face databases, the CASIA database. The proposed system provides recognition accuracy that is resulted in to an efficient 3D face recognition system.
\end{abstract}

\section{Keywords}

Fuzzy-Neural Network (FNN), 3D-Face Recognition, Depth Map, Moment Invariants.

\section{INTRODUCTION}

A facial recognition is the process of automatically identifying or verifying a person from a digital image or a video frame and has been a recent area of research. Nowadays face recognition is becoming more and more necessary due to increasing demand of high- security systems [1]. To make the face recognition more efficient, the robustness, higher recognition rates, tolerance for ambiguity and uncertainty should be considered as essential factors for implementation. A new mathematical approach known as soft computing (SC) is emerging that shows promise in dealing with such problems and models human behavior. The soft computing techniques like Neural Network, Fuzzy Systems and Genetic Algorithms are used to implement the intelligent systems that possess human like expertise within a specific domain. They adapt themselves and learn to perform better in changing environments. But an individual soft computing technique has certain limitations i.e. while neural networks are good at recognizing patterns; they are not good at explaining how they reach their decisions. Fuzzy logic systems, which can reason with imprecise information, are good at explaining their decisions but they cannot automatically acquire the rules they use to make those decisions [2]. One of the solutions to overcome the lack of the transparency in neural networks is to combine them with fuzzy systems. We propose a novel hybrid face recognition which uses neural-fuzzy system.

The resultant hybrid system overcomes the limitations of both the techniques. The capability of fuzzy system provides the insight to the user about the knowledge embedded within the neural network. The Fuzzy-Neural Network combines the advantages of neural network and fuzzy systems. The advantage of using neural network is its capability to train itself by learning from datasets but the system cannot be easily interpreted, whereas in fuzzy systems the knowledge representation is explicit[3], i.e. verification and optimizations are very easy and efficient.

In this proposed system the depth maps; two dimensional matrices containing depth information are generated from the 3D face images. These depth maps are the input for the fuzzyneural network. The classification and recognition is carried out using the trained and test images .In this proposed system the neural network is used to tune membership functions of fuzzy system that are employed as decision-making systems for face recognition system. The CASIA 3D face database is used for this face recognition system. Initially only neural network was used for the face recognition but the hybrid recognition method outperformed and generated promising results.

\section{RELATED WORK}

The hybrid information system containing combinations of soft computing methods are being used in recent face recognition systems. The soft-biometrics is the biometric using soft computing technique. In [1] the concept of softbiometrics is explained where in the process of biometrics and the significance of use of soft computing techniques is discussed. The example of signature verification is used as the application of soft-biometrics. Combination of two techniques results in to promising biometric system with benefits of both the systems. The hybrid intelligent systems are widely being used by the researchers for the complex process like face recognition. In [2] the hybrid intelligent system uses ant colony optimization algorithm and enhanced genetic algorithm to implement the static face recognition system in a multifaceted condition. In [3] the authors have implemented fuzzy-neural system and investigated the benefits of combining neural networks and fuzzy logic in this system the PCA is used for dimension reduction. Reference [4] covers the basics of neural-fuzzy applications in computer vision. In this article the authors have explained the use of neural-fuzzy systems for problematic and complex computer vision applications using neural-fuzzy systems. The implementation of [5] has proposed the hybrid system which enables the conversion between fuzzy systems and neural network augmenting the advantages of both the systems. In [6] the survey on neural-fuzzy system is available in detail, as well as overall process of rule generation, process of hybridization and incorporation of fuzzy system in neural network is described. Very recently the credit scoring model was proposed in [7], in which the network modeling issues were considered, and the soft computing approaches which can be applicable in credit models are reviewed which can be applicable in general applications also. In [8] the authors have described a face recognition technique that uses eyes, mouth, and nose locations as facial features, the neural classifiers considers varying degrees of reliability for each feature. The decisions of individual classifiers are then combined using a fuzzy information fusion technique. The researchers are 
increasingly using soft computing methods for hybrid intelligent systems, but from current research it is not clear that neural-fuzzy outperform other competing techniques because of its slow learning process. Still the limitations of individual soft computing technique have been a central driving force behind the creation of intelligent hybrid systems where two or more techniques are combined in a manner that overcomes the limitations of individual techniques.

\section{3D FACE DATABASE}

Face recognition is a well known biometric technique specially used for human identification and mostly applicable in security applications, user identification, and enrollment /authentication systems. Generally most of the applications use the 2D face recognition systems, but the performance of these systems badly affects due to changes in various factors like pose, expression, lighting conditions, etc. Nowadays 3D face recognition has become an ever-growing and promising biometric technique because of its robust features [9].

The 3D facial data can provide a promising way to understand the characteristics of the human face in 3D domain, and has potential possibility to improve the performance of the recognition system. And more and more researchers focus on $3 \mathrm{D}$ face recognition in the past few years. However, since the $3 \mathrm{D}$ cameras are not as common as $2 \mathrm{D}$ cameras, it is expensive to build a public 3D face database, which brings the difficulty to validate the proposed methods in a uniform platform.

CASIA 3D face database is one of the important 3D databases. This database contains $2 \mathrm{D}$ as well as 3D face images and it is created by Chinese Academy of Sciences.

The images were collected using a non-contact 3D digitizer, Minolta VIVID 910, working on Fast Mode. This database contains 123 subjects, with each subject having 33 images. The database is divided into three subsets, that is, the training set, the gallery set and the probe set. The images in this database are VRML images. These are also referred to as world files having extension wrl. The CASIA 3D face database contains the images with various head poses, but for this proposed system the images with frontal pose are considered as the probe images.

\subsection{Image Representations and Feature Extraction}

The Images are represented in two forms; depth maps and mesh images. Dept map representation handles the illumination effect considerably whereas the mesh images are used for extracting the moment invariants.

PCA on Depth Maps

The most popular approach in 3D face recognition systems has been to convert the $3 \mathrm{D}$ point cloud information into $2 \mathrm{D}$ depth images (range images). This conversion operation is needed because the 3D data may not correspond to a regular grid. While the 2D data is more familiar to work with, the loss of genuine information must be accounted for. Once the depth image is formed, one can treat the 3D face recognition problem as simply a 2D face recognition problem and wellknown 2D face recognition techniques such as PCA, LDA, and ICA can be employed on the 2D depth maps.

The depth maps are used as 2D representation of 3D image, containing the depth information of each point in the 3D image. It is simply an image with depth information as shown in Fig. 1-b. In other words, a depth map is an array of numbers where the numbers quantify the distances from the focal plane of the sensor to the surfaces of objects within the field of view. The closest point has the highest value. And therefore the closest point appears as white and farthest point appears as black giving the gray level values to the between points. The depth images have some advantages over 3D images [12]. The most important one is that the depth maps are robust to the change of illumination and color because the value on each point represents the depth value which does not depend on illumination or color [14].

The 3D face images contain highly accurate data but it is not feasible to process the large amount of facial data. Because the face model of each subject model has different vertices. The processing of the large data results into the expensive computation.

Face recognition systems based on 3D facial depth information improves the accuracy and robustness. But have not been addressed thoroughly. Only a few works on the use of depth map have been reported. The fusion of depth map and texture map is used in [10] where Fisher face and FaceIt techniques are used and FaceIt method outperformed on fusion of depth and texture information. The fusion of depth map and curvature information is used in [11].

In this proposed system the depth maps containing depth information are generated from the available 3D face images. The training data set contains the normalized depth maps of all the subjects which are selected as the candidates for training datasets. Since our main objective is to evaluate the results for handling the illumination variations, all frontal images with varying light are considered as the candidates for the face recognition.

As shown in figure 1a the frontal images from the CASIA database are considered as probe images. For each probe image the depth image is generated (Figure 1b) The normalization process is carried out to generate the normalized depth image as shown in figure 1c.
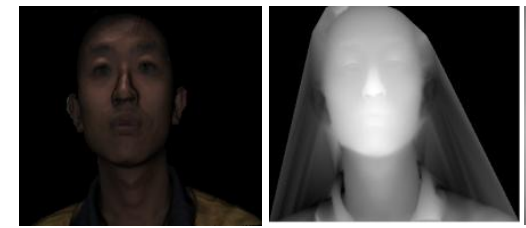

Figure 1: a) 3D Face Image b) Depth map c) Normalized Depth Map

The principal components analysis is applied on the depth maps of the face images for dimension reduction. The projections of depth maps are used as features for face recognition

\subsection{Moment Invariants of 3D Mesh Model}

Meshes are widely used to represent 3D models. The Features which are helpful for face recognition can efficiently be calculated from the mesh representation. The mesh models generated from the 3D face images are shown in figure 2 .

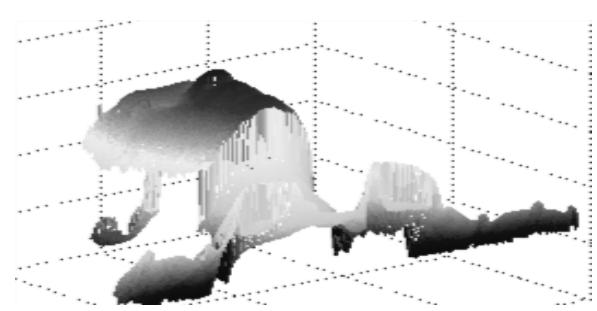

Figure 2: Mesh Representation 
From the mesh images the moment invariants are generated and these moment invariants are used as rotation invariant features for face recognition.

Moments are a measure of the spatial distribution of 'mass' of a shape. In the case of binary digital datasets, this is the distribution of pixels (in 2D) and voxels (in 3D) of a shape. It is possible to compute moment invariants of 3D point distributions (i.e. voxels) that are invariant to translation and rotation, in the same manner as 2D moment invariants.

Let $f(x, y, z)$ be a local continuous density function. For example, this can be 1 inside voxel belonging to an object and 0 in free space.

In classical recognition problems, the concept of moments is used extensively. The moments of a 3D mesh model can be defined as:

$$
\begin{aligned}
& M_{p q r}=\iiint_{-\infty}^{\infty} x^{p} y^{q} z^{r} f(x, y, z) d x d y d z \\
& \quad \mathrm{x}, \mathrm{y}, \mathrm{z} \in\{-1,1\}
\end{aligned}
$$

Where $\mathrm{f}(x, y, z)$ is an indicator function; and $p, q, r$ are the orders of the moment.

If the density is piece wisely continuously and bounded in a finite region in 3D Euclidean space, then moments of all order exists.

Where $\mathrm{n}=\mathrm{p}+\mathrm{q}+\mathrm{r}$, is the order of the moment,

$(x, y, z)$ are the coordinates of each voxel,

and $\mathrm{f}(x, y, z)$ are the statistics representing the level of activation of each voxel inside the ROI.

3D moments provide the general 3D shape information of $\mathrm{f}(x, y, z)$. Therefore, metrics invariant to rotational, translational, and scaling artefact are required. In this proposed system, 3D moment invariants were used to ensure that only true shape differences in the activation are captured, independent to the particular co-ordinate system used.

The centroid of the 3D mesh model can be obtained easily from the zeroth and first-order moments as shown below

$$
x=\frac{M_{100}}{M_{000}}, \quad y=\frac{M_{100}}{M_{000}} \quad z=\frac{M_{100}}{M_{000}}
$$

Using the centroid in the moment calculation below gives translation invariance.

The central Moments are defined as

$M_{p q r}=\iiint_{-\infty}^{\infty}\left(x-\bar{x}^{p}\left(y-\bar{y}^{q}\left(z-\bar{z}^{r} f(x, y, z) d x d y d z\right.\right.\right.$

Scale invariance can be obtained by normalizing the moments as follows:

$n_{p q r}=\frac{m_{p q r}}{m_{000}^{\frac{p+q+r}{2}+1}}$
To obtain rotational invariance, the 3D moments need to be summed in a certain fashion as given in equations (4) to (10) The Seven invariants were generated which are based on 2 nd order moments as derived in [15].

$$
\begin{aligned}
I_{1}= & M_{200}+M_{010}+M_{002} \\
I_{2}= & M_{200} M_{020}+M_{200} M_{002}+M_{020} M_{002}-M_{101}^{2}-M_{110}^{2}- \\
& M_{011}^{2} \\
I_{3}= & M_{200} M_{020} M_{002}-M_{002} M_{110}^{2}+2 M_{110} M_{101} M_{001}- \\
& M_{101}^{2}-M_{011}^{2}-M_{020} M_{101}^{2}-M_{200} M_{011}^{2}
\end{aligned}
$$

The remaining four higher order invariants used are based on moment tensor contraction $\left(\mathrm{B}_{3}\right.$ to $\left.\mathrm{B}_{6}\right)$

$$
\begin{aligned}
I_{4}= & M_{200}+M_{020}+M_{002}+3 M_{210}+3 M_{201}+3 M_{120}+ \\
& 6 M_{111}+3 M_{102}+3 M_{012}+3 M_{021} \\
I_{5}= & M_{400}+M_{040}+M_{004}+4 M_{310}+4 M_{301}+6 M_{220}+ \\
& 12 M_{211}+6 M_{202}+4 M_{130}+12 M_{121}+12 M_{112}+ \\
& 4 M_{103}+\quad 4 M_{031}+6 M_{022}+4 M_{013} \\
I_{6}= & M_{500}+M_{050}+M_{005}+5 M_{410}+5 M_{401}+10 M_{220}+ \\
& 20 M_{211}+10 M_{302}+10 M_{230}+30 M_{221}+30 M_{212}+ \\
& 10 M_{203}+20 M_{131}+30 M_{122}+20 M_{113}+5 M_{104}+ \\
& 5 M_{041}+10 M_{032}+10 M_{023}+5 M_{014} \\
I_{7}= & M_{600}+M_{060}+M_{006}+6 M_{510}+6 M_{501}+15 M_{420}+ \\
& 30 M_{411}+15 M_{402}+20 M_{330}+60 M_{321}+60 M_{312}+ \\
& 20 M_{303}+15 M_{240}+60 M_{231}+90 M_{222}+60 M_{213}+ \\
& 15 M_{204}+6 M_{130}+30 M_{141}+60 M_{132}+60 M_{123}+ \\
& 30 M_{114}+6 M_{105}+06 M_{051}+15 M_{042}+20 M_{033}+ \\
& 15 M_{024}+6 M_{015}
\end{aligned}
$$

More number of higher order invariants can be derived using similar procedures to increase the number of features which might boost discriminative power of the feature vector. However, the chosen set of 3D moment invariants have provided adequate shape discriminative capability for the purposes of rotation invariant face recognition.

\section{METHODOLOGY}

Face recognition is an important biometric application among many applications such as personal identification, access to high security areas, human-machine interactions but not limited to these. The main advantage of face recognition as a biometric system is its throughput, convenience and noninvasiveness. 3D facial information has become an important biometric modality due to its ability in handling challenging problems that occurs due to illumination changes and facial pose variations.

In the proposed system the FNN (fuzzy neural network) is used for feature extraction and classification. Initially the fuzzy system is used for feature extraction which assigns membership function to the feature and these features with membership function are provided to neural network to match the probe image with the images in the training dataset. The outline of the proposed system is shown in the fig.3. The depth maps are robust to color and illumination.

Therefore the problems of illuminations are handled by considering the normalized depth map which is taken as the input images. Before giving the input to the fuzzy-neural network the PCA is applied on the depth maps for dimension reduction.

PCA approach is as follows: 
- Construct a covariance matrix of a training sample set (depth maps);

- Generate eigenvectors using PCA. The first kth eigenvectors span an eigen subspace;

- Project a probe sample into this subspace for dimension reduction and eigen representation.

On other hand the mesh images are generated from the 3D face images and the 3D moment invariants are extracted as features which are rotation invariant

The combination/fusion of classifier is employed to perform the classification. The system will conclude on Match or NonMatch based on extracted features and the threshold.

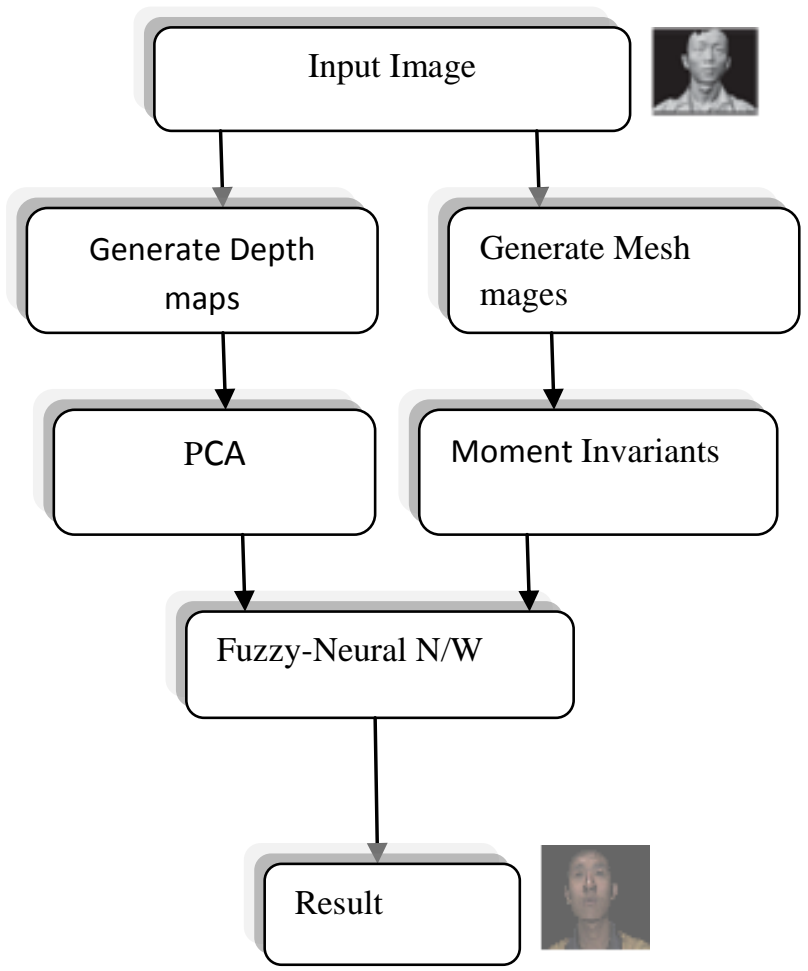

Figure 3. Outline of proposed face recognition system

\subsection{Fuzzy Neural Network}

There have been numerous successful research experiments on using learning algorithms for constructing intelligent applications. These approaches are capable of successfully working in the ill-defined and changing environments.

Neural networks have established themselves as a serious alternative to traditional statistical models and many studies have concluded that neural networks outperformed statistical methods in terms of classification accuracy.

In last decade the fuzzy logic and neural networks are being widely used. The fuzzy logic provides an inference mechanism in uncertain conditions. It converts the crisp function in to fuzzy output, whereas the neural network offers the learning capability to the system. The capability of fuzzy system provides the insight to the user about the knowledge embedded within the neural network. The Fuzzy-Neural Network combines the advantages of neural network and fuzzy systems. The advantage of using neural network is its capability to train itself by learning from datasets but the system cannot be easily interpreted, whereas in fuzzy systems the knowledge representation is explicit, making the recognition easy and efficient.
The Fuzzy Neural network incorporates the modeling and learning capabilities of neural network into human reasoning capability of fuzzy logic. The resultant hybrid system resembles an adaptive system with sensory and cognitive components.

\subsubsection{Steps Involved in FNN}

In the fuzzy neural network fuzzy rules are determined by the data. The FNN has four layers [13]. The Input layer, Fuzzification layer, Inference layer and Defuzzification layer (fig. 3). In the input layer the ' $N$ ' input neurons are inputted for fuzzification. The fuzzification process generates the ' $R$ ' rules which are in turn taken as the input in inference layer.

- $\quad$ Fuzzy membership generation.

- Indentify the significant input variable from the input space using membership functions and the inference curves.

- Determine the fuzzy rules, select the number of rules and select the appropriate rules from the possible rules.

- Set the initial weights to determine the membership function.

- In fuzzification layer, every neuron is represented by the membership function $\left(\mu_{\mathrm{ij}}\right)$

Here $\mu_{\mathrm{ij}}$ is the membership function of the $\mathrm{i}^{\mathrm{ih}}$ input variable corresponding to $\mathrm{j}^{\text {th }}$ rule.

- Train the network using membership functions.

- Drop unnecessary rules and neurons.

In the proposed system the desired output vector will be the class to which the input image belongs. In the conventional systems the number of nodes in the output layer corresponds to the number of classes in the task to be performed where the desired output vector is assigned the value 1 , and other classes are assigned the value 0 ; this is called a crisp desired output. In the face recognition applications, however, the data are generally ill-defined, with overlapping. That is, there are chances to have patterns with non-zero similarity to two or more pattern classes. The fuzzy neural network in the proposed face recognition system handles this multiple similarity or membership value. Figure 4 represents the architecture of the fuzzy neural network.

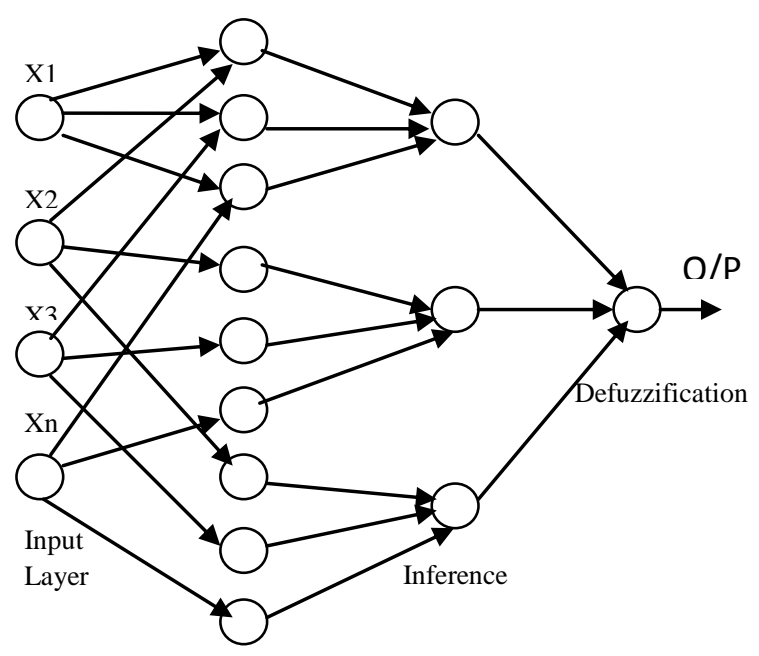

Fuzzification

Figure 4: Architecture of FNN 
The input layer reads the probe image and the membership function at fuzzification layer performs the classification to determine the closest class which belongs to. Inference layer generates the output to recognize the input face image.

The feature vectors are obtained using depth maps of the face images. Then, the output vectors obtained from that step must be fuzzified. The feature vector is compared with the class mean vector. That is the more a feature vector approaches towards the class mean vector, the higher is the fuzzy value. When the difference between both vectors increases, the fuzzy value approaches towards 0 . The patterns whose class is less certain have lesser role in weight adjustment. The pseudo code for this proposed system can be given as follows.

- Let there be ' $\mathrm{k}$ ' classes

- $\quad x$ ' be the feature vector to be recognized

- $\quad \mathrm{f} 1, \mathrm{f} 2, \mathrm{f} 3$....fk are the fuzzy set membership functions that will decide whether the vector belongs to the class ' $\mathrm{m}$ ' or not.

- Initially input the significant input-vectors: $\mathrm{x} 1, \mathrm{x} 2 \ldots \mathrm{xn}$ containing PCA of depth map representations and moment invariants of mesh models of face images.

- If there are ' $n$ ' test images, ' $n$ ' sets of fuzzy rules will be generated

- Calculate weights using the difference between the desired output and actual output.

- In defuzzification step; use threshold value to convert the membership value to either ' 0 ' or ' 1 '

- Declare the class with highest membership value as the class containing the test image.

In this paper we have introduced hybrid system with the implementation of fuzzy mathematics in neural networks for face recognition. Initially the same experiment was carried out using conventional neural network. The Fuzzy Neural network has outperformed because of the additional capability which has been provided to neural network by embedding the fuzzy logic in it. The varying size of training data also affects the performance of conventional neural network system. As per the observations, with the increase in number of training images, the efficiency of FNN also increases but during the experiments on FNN no false rejection was observed. The performance evaluation of the proposed system is shown in the table1. The use of depth images of 3D face images as input images has considerably handled the varying lighting effects. In [14] only frontal images were used and the experiments were carried out to study the performance of fuzzy neural network on depth map and it was found that the use of depth map effectively handles the illumination variations. In this paper extension to the work [14] is proposed and used moment invariants on frontal as well as non-frontal images to develop the rotation invariant face recognition system. Table 1 .shows the significant results by comparing the three approaches; FNN-VLE-F (Fuzzy Neural Network with varying lighting effects on frontal images), FNN-VLE-N (Fuzzy Neural Network with varying lighting effects on Non-frontal images), FNN-VRL-FN (Fuzzy Neural Network with varying Rotation and Lighting effects on Frontal \& Non-frontal images). The graphical representation is shown in figure5.
Table 1: Observations

\begin{tabular}{|c|c|c|c|}
\hline \multirow{2}{*}{\multicolumn{2}{|c|}{ Methods used }} & \multicolumn{2}{|c|}{ Performance details } \\
\hline & & \multirow{2}{*}{$\begin{array}{l}\begin{array}{c}\text { No of training } \\
\text { images }\end{array} \\
10 \\
\end{array}$} & \multirow{2}{*}{$\begin{array}{l}\text { Performance } \\
95 \% \\
\end{array}$} \\
\hline \multirow{6}{*}{$\begin{array}{l}\text { FNN- } \\
\text { VLE }\end{array}$} & \multirow{3}{*}{$\begin{array}{l}\text { Frontal } \\
\text { Images }\end{array}$} & & \\
\hline & & 25 & $96 \%$ \\
\hline & & 50 & $98 \%$ \\
\hline & \multirow{3}{*}{$\begin{array}{l}\text { Non- } \\
\text { Frontal } \\
\text { Images }\end{array}$} & 10 & $88 \%$ \\
\hline & & 25 & $88 \%$ \\
\hline & & 50 & $87 \%$ \\
\hline \multirow{3}{*}{\multicolumn{2}{|c|}{$\begin{array}{l}\text { FNN-VRL } \\
\text { (On frontal+ Non- } \\
\text { frontal images) }\end{array}$}} & 10 & $98.5 \%$ \\
\hline & & 25 & $98.9 \%$ \\
\hline & & 50 & $99 \%$ \\
\hline
\end{tabular}

Thus the hybrid intelligent system combines the positive traits of both the systems to incorporate the expressive power of the rules, transparency of view taken in the $\mathrm{NN}$ algorithm where they can mimic the behaviour of the human being.

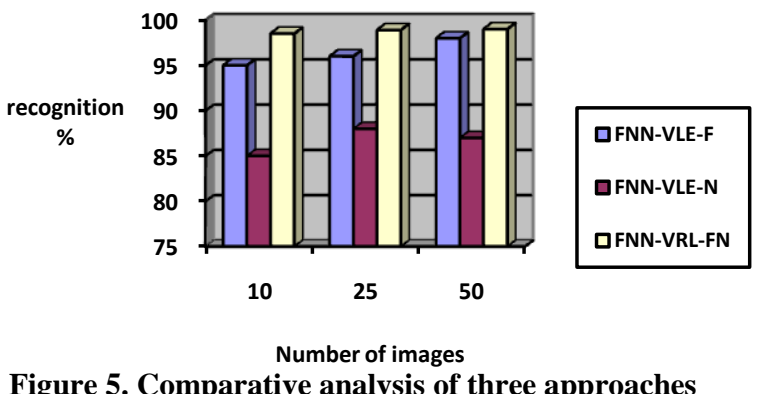

Thus from Table 1 and Figure 5 it is found that the performance of the proposed system is consistent on both the frontal as well as Non-frontal images.

\section{CONCLUSION}

To improve the performance of the face recognition systems, many face recognition applications have been developed using the combination of two or more soft computing techniques. Fuzzy Neural Network offers the precision and learning capability of neural networks, and yet is easy to understand like fuzzy systems. The proposed 3D face recognition system has used the hybrid fuzzy neural network to incorporate the thinking and reasoning capability. An integration of the merits of neural and fuzzy approaches enables one to build more intelligent decision-making systems. Also the combination of features generated from depth-maps and mesh images have resulted into the implementation of automated 3D face recognition system which is robust to illumination and rotation variations.

\section{REFERENCES}

[1] Katrin Franke , Javier Ruiz-del-Solar , Mario Koppen, "Soft-Biometrics: Soft-Computing for BiometricApplications," Dept. of Pattern Recognition, Fraunhofer IPK, Berlin, Germany. 
[2] S.Venkatesan and Dr.S.Srinivasa Rao Madane, "Experimental Research on Identification of Face in a Multifaceted Condition with Enhanced Genetic and ANT Colony Optimization Algorithm", International Journal of Innovation, Management and Technology, Vol. 1, No. 5, December 2010, ISSN: 2010-

[3] Nina Taheri Makhsoos, Reza Ebrahimpour , Alireza Hajiany," Face Recognition Based on Neuro-Fuzzy System", IJCSNS International Journal of Computer Science and Network Security, VOL.9 No.4, April 2009.

[4] Henry O. Nyongesa, Paul L. Rosin, "Neural-Fuzzy Applications in Computer Vision", Editorial, Journal of Intelligent and Robotic Systems 29: 309-315, 2000.

[5] Ryusuke Masuoka et al.,Neurofuzzy system-Fuzzy Inference Using a Structured Neural Netwok, Procedings of the international conference on Fuzzy Logic and neural Network, Japan, July 20-24,1990,pp 173-177

[6] Sushmita Mitra, Member, IEEE, and Yoichi Hayashi, Senior Member, IEEE, " Neuro-Fuzzy Rule Generation: Survey in Soft Computing Framework", IEEE Transactions On Neural Networks, VOL. 11, NO. 3, May 2000

[7] Adel Lahsasna, Raja Noor Ainon, and Teh Ying Wah, "Credit Scoring Models Using Soft Computing Methods: A Survey", The International Arab Journal of Information Technology, Vol. 7, No. 2, April 2010

[8] Mirhosseini, A. R., Yan, H., Lam, K. M., and Pham, T.: Human face image recognition: An evidence aggregation approach, Computer Vision Image Understanding 71(2) (1998), 213-230.

[9] Nita M. Thakare,V.M. Thakare," Representation and Pre-processing Techniques for Illumination Invariant 3D Face Recognition", ICWET'11, February 25-26, 2011, ACM 978-1-4503-0449-8/11/02

[10] Chiraz BenAbdelkadera, Paul A. Griffin, Comparing and combining depth and texture cues for face recognition, Image and Vision Computing 23 (2005) 339-352

[11] Gaile G. Gordon, Face Recognition Based on Depth and Curvature Features, TASC 55 Walkers Brook Drive Reading, MA 01867

[12] Chuanjun Wang, Xiamu Niu,Wenwei Lu, Jianren Gong "A Hybrid Method to Build a Canonical Face Depth Map" , International Journal of Digital Content Technology and its Applications. Volume 5, Number 5 , May 2011

[13] Neuro Fuzzy Control Systems, edited by M.R. Kaimal, S. Dasgupta, M. Harisankar.

[14] N.M.Thakare,V.M. Thakare," An Innovative Hybrid Approach to Construct Fuzzy-Neural Network for 3D FaceRecognition System" , IEEE proc., 11th International Conference on Hybrid Intelligent Systems HIS 2011.D O I: 10.1109/HIS.2011.6122149 , 2011 , PP. $463-467$

[15] F. A. Sadjadi and E. L. Hall, "Three-Dimensional Moment Invariants," IEEE Trans. Pattern. Anal. Machine Intell., vol. PAMI-2, pp. 127-136, Mar 1980. 